\title{
Rechtsgeschichte
}

www.rg.mpg.de

http://www.rg-rechtsgeschichte.de/rg12

Zitiervorschlag: Rechtsgeschichte Rg 12 (2008)

$\operatorname{Rg} 12_{2008}$

$176-182$

http://dx.doi.org/10.12946/rg12/176-182

\section{Wolfram Brandes}

\section{Bilder und Synoden}




\section{Bilder und Synoden*}

Der byzantinische Bilderstreit des 8 . und 9. Jahrhunderts ist ein unerschöpfliches Thema, das alljährlich mehrere Bücher und noch mehr Aufsätze generiert. Und gelegentlich schafft er es sogar - wenn auch nur en passant -, in den Feuilletons der großen Tageszeitungen Erwähnung zu finden. So etwa Anfang 2006, als (rechtslastige) Journalisten in Dänemark meinten, Muslime mit Muhammadkarikaturen provozieren zu müssen - was ihnen bekanntlich ja auch gelang. Allerdings diente der mittelalterliche Streit über die Berechtigung der Verehrung heiliger Bilder lediglich als pseudogelehrtes Ornament der geführten Debatte. Ob dies dazu führte, dass irgendjemand zu dem kurz zuvor erschienenen Band von Thümmel über die Synoden zur Bilderfrage im 7. und 8. Jh. griff, um sich weiter über diesen Themenkomplex zu informieren, vermag der Rezensent natürlich nicht zu sagen. Auszuschließen ist es nicht. Und sicher hätte man genügend Informationen gefunden, um sich ein Bild vom Bilderstreit zu machen. Man hätte erfahren können, dass dieser byzantinische Gelehrtenstreit - um einen solchen handelt es sich in erster Linie - nichts mit dem islamischen Bilderverbot zu tun hatte, wie man früher oft meinte.

Dennoch kam dieses Buch gerade recht, um diejenigen, die sich über die byzantinische Auseinandersetzung um den Ikonenkult bzw. die Verehrung von Heiligenbildern informieren möchten, mithin näher an einer wesentlichen Eigenheit der sog. "orthodoxen " Kirchen interessiert sind, einen sicheren Leitfaden an die Hand zu geben, mit dessen Hilfe sie sich durch das Dickicht der komplizierten und widersprüchlichen Quellen sowie der ausufernden Sekundärliteratur tasten können.
Der Verf. hat sich seit mehreren Jahrzehnten mit der Thematik befasst, wie eine Vielzahl einschlägiger Publikationen eindrucksvoll belegt. ${ }^{\mathrm{I}}$ Nun fasst er in diesem Band diese Forschungen zusammen. Entstanden ist ein "altertümliches « Buch - im besten Sinne des Wortes. Einen gewissen naiven Charme in methodischtheoretischer Hinsicht kann man dem Band nicht absprechen (»Mir geht es um ein neues Wahrnehmen und differenzierendes Lesen der Quellen. Die Texte sollen um Sprechen kommen.« [XXII]). Man kann ihn - mit gutem Recht natürlich auch als theoriefrei bezeichnen, was eine Auseinandersetzung mit methodischen oder irgendwie gearteten theoretischen Ansätzen überflüssig erscheinen lässt. Dass hier selbstverständlich keine »wertfreie " Darstellung vorliegt (vorliegen kann), braucht nicht weiter vertieft zu werden; das hört jeder Geschichtsstudent schon im Proseminar. "Mein Ziel ist es, ohne Voreingenommenheit zu klären, was war « (XXI). Das ist natürlich homöopathisch verdünnter Ranke. Die hier geäußerten Ansichten und methodischen »Programme « basieren auf einem 2002 erschienenen Aufsatz, ${ }^{2}$ der sich kritisch mit einer neueren Publikation zur ersten Phase des Bilderstreites auseinander setzte. ${ }^{3}$ Insbesondere waren die zitierten Worte gegen Paul Speck († 2003) gerichtet, der ebenfalls seit Jahrzehnten den byzantinischen Bilderstreit untersuchte. Speck befleißigte sich einer radikalen destruierenden Methodik, die letztlich zeigen sollte, dass fast alle Texte, die wir über den Bilderstreit besitzen, spätere Konstrukte sind. Allerdings ging er, wie sich das oft bei einem innovativen Forschungsansatz beobachten lässt, entschieden zu weit. Sein Entwurf des byzantinischen Literaturbe-
* Hans Georg Thümmel, Die Konzilien zur Bilderfrage im 8. und 9. Jahrhundert (Konziliengeschichte, hg. von W. Brandmüller, Reihe A: Darstellungen), Paderborn, München, Wien, Zürich: Ferdinand Schöningh 2005, XXIV, 3 I9 S.; ISBN 3-506-7I 374-4

I Hans Georg Thümmel, Bilderlehre und Bilderstreit. Arbeiten zur Auseinandersetzung über die Ikone und ihre Begründung vornehm- lich im 8. und 9. Jahrhundert, Würzburg I99I; DERs., Die Frühgeschichte der ostkirchlichen Bilderlehre. Texte und Untersuchungen zur Zeit vor dem Bilderstreit, Berlin I992; DERs., Karpoi. Ausgewählte Aufsätze zu Patristik Philosophie - Christliche Kunst (1966-2004), Frankfurt am Main 2007.

2 Hans Georg Thümmel, Der byzantinische Bilderstreit bis zum
Konzil von 754 , in: Annuarium Historiae Conciliorum 34 (2002) I6-56, hier bes. I 8-20.

3 Die ikonoklastische Synode von Hiereia 754. Einleitung, Text, Übersetzung und Kommentar ihres Horos, besorgt von ToRsten Krannich, Christoph Schubert, Claudia Sode, Tübingen 2002. 
triebs des 9. und I0. Jahrhunderts nach dem Vorbild eines Orwellschen "Ministery of Truth « nahm bisweilen absurde Züge an. ${ }^{4}$ Vom erhaltenen Schrifttum des byzantinischen Bilderstreites konnte kaum ein Text die Specksche Radikalkritik überstehen. Thümmel verzichtet im vorliegenden Band auf eine Auseinandersetzung mit den Thesen von Speck. Es blieb ihm auch kein anderer Ausweg, denn sonst wäre sein Buch unlesbar und hätte einen hermetischen Charakter angenommen, den nur wenige Leute in der Welt - eben die, welche die zahllosen Kontroversen zwischen Speck und Thümmel seit mehr als zwei Jahrzehnten verfolgt haben - hätten begreifen können.

Es ist bezeichnend für den Zustand der heutigen Byzantinistik, insbesondere in Deutschland, dass sie jegliche theoretische Reflexion über ihren Gegenstand, ihre Methoden usw. wie der Teufel das Weihwasser meidet. Das mag man beklagen. Man kann jedoch auch froh darüber sein. Denn auf der einen Seite präsentiert sie sich wie der Quastenflosser (Coelacanthiformes) der modernen Biologie den modernen Geistes- und Sozialwissenschaften als ein höchst interessantes Relikt. Um nicht zu übertreiben: Es gibt natürlich auch in der byzantinistischen Forschung moderne theoretische Ansätze, doch die hat Thümmel (wie auch Speck) konsequent ignoriert. $^{5}$

Auf der anderen Seite führt dies dazu, dass der Verfasser eine präzise Zusammenfassung der einzelnen Vorgänge sowie der Ansichten der am theologischen Diskurs beteiligten Persönlichkeiten präsentiert. Dies mag auch an dem Charakter der Reihe "Konziliengeschichte «, herausgegeben von Walter Brandmüller, dem Oberhistoriographen des Vatikan, liegen. Es fehlt eine wirkliche Einsicht (geschweige denn Darstellung) in die historischen (insbesondere wirtschaftlichen und sozialen, aber auch verwaltungsgeschichtlichen usw.) Zeitumstände und Hintergründe. Der Bilderstreit begleitete den Aufstieg des byzantinischen Staates und seiner Gesellschaft, nachdem im 7. Jahrhundert durch den fast unaufhaltsamen Ansturm der Araber eine im höchsten $\mathrm{Maß}$ krisenhafte Periode zu überwinden war. Der Bilderstreit ist also nicht Ausdruck einer Krise, wie man bis heute immer wieder lesen kann (nicht aber im vorliegenden Buch, das diese Frage übergeht). ${ }^{6}$ Vielmehr ist er ein Aspekt eines teilweise heftig geführten Diskurses über die weitere gesellschaftliche, staatliche und ideologische Entwicklung des nunmehr zum mittelalterlichen byzantinischen Staat mutierten Römischen Reiches. Dies war ein in weltgeschichtlicher Hinsicht höchst bedeutender Vorgang, dessen » Begleitmusik « der sog. Bilderstreit war: Er stellte einen Aspekt des Ringens des nunmehr konsolidierten Byzantinischen Reiches um seine neue Identität dar. Zwar sah man sich ungebrochen als "Römer « (Rhomaioi), doch grenzt es an Hybris, zu unterstellen, die Byzantiner des 8. oder 9. Jahrhunderts hätten nicht begriffen, dass ihr Römertum nichts mehr mit dem eines Augustus, Konstantin des Großen oder Justinian zu tun hatte. Alles war anders geworden; geblieben war die fiktive Hülle, eher Schablone, eines »Römischen Reiches", das weder in geographischer, kultureller (das Griechische hatte sich umfassend durchgesetzt), verwaltungstechnischer (von den einst sakrosankten Institutionen des Imperium Romanum war außer Namen kaum etwas geblieben) noch in politischer Hinsicht einen Vergleich mit dem wirklichen Römischen Reich, insbesondere in seiner christianisierten Form seit Konstantin dem Großen, aushalten konnte. Der neue byzantinische Staat brauchte eine eigene, passende Identität, die so viel wie möglich der römischen (christlichen)
4 Bibliographie in Novum Millennium. Studies ... dedicated to Paul Speck, Aldershot 200I, XI-XIX. 5 Siehe z. B. Mischa Meier, Das andere Zeitalter Justinians. Kontingenzerfahrung und Kontingenzbewältigung im 6. Jahrhundert n. Chr., Göttingen 2003, hier bes. 528-559; John Haldon, Some Remarks on the Background to the Iconoclastic Controversy, Byzantinoslavica 38 (I977) I6I-
I84; Peter Brown, A Dark-Age Crisis: Aspects of the Iconoclastic Controversy, English Historical Review 87 (I973) I-34; Michael McCormick, Textes, images et iconoclasme dans le cadre des relations entre Byzance et l'Occident Carolingien, in: Settimane Spoleto 4I (I994) 95-I 58 u. a.

6 Es ist deshalb besonders absurd, wenn Adolf Martin Ritter,

Fragen zu H. G. Thümmels Syn- these »Die Konzilien des byzantinischen Bilderstreits «, in: Zeitschrift für antikes Christentum Io (2006/2007) 195-206, Thümmel vorwirft, er habe den Krisencharakter der Zeit nicht genügend beachtet. 
Tradition verpflichtet sein sollte, aber auch den neuen Bedingungen entsprechen musste, die so grundsätzlich anders waren als die des 4 . bis 6. Jahrhunderts. Das waren komplexe und divergierende Entwicklungen, die alle Bereiche der Gesellschaft erfassten - nicht nur die Praxis des christlichen Kultus und der damit zusammenhängenden theologischen Debatten, so heftig diese auch geführt wurden.

Auf derartige (oder andere) Überlegungen verzichtet der Verfasser. Bei ihm erscheint die Auseinandersetzung um den Bilderkult als ein langfristiges innerkirchliches Problem, das dann urplötzlich (im Jahre 726) vom regierenden Kaiser aufgegriffen wurde (warum?) und sich in den folgenden Jahren zu einem heftigen Konflikt steigerte. So jedenfalls das Bild der erhaltenen Quellen, die z. T. erheblich später entstanden als die Vorgänge, die sie schilderten und dadurch konstruierten!

Leider hat Thümmel auch neuere Ansätze ignoriert, die die ausgetretenen Pfade der traditionellen Forschung verlassen und neue Gedanken und Deutungsmuster ins Spiel der Forschung zur Bilderfrage und ihrer historischen Dimension gebracht haben. Dass diese wichtige Periode der byzantinischen Geschichte, in der die Grundlagen für den kometenhaften Wiederaufstieg des Reiches nach seiner Krise gelegt wurden, bis heute primär unter dem Gesichtspunkt des Bilderstreites gesehen wird, ist letztlich das Resultat der Geschichtsmanipulationen der »Sieger «, der Bilderfreunde, die bis heute das Gesicht der orthodoxen Kirchen mit ihrem extensiven Ikonenkult prägen. Nach 843 , dem Jahr des endgültigen Sieges der Bilderverehrer, kam es zu einer Geschichtsrevision, die allerdings nicht die von Speck (s. o.) intendierten Orwellschen Dimensionen annahm. Im Ergebnis blieben (fast) allein bilderfreundliche Texte erhalten. Die Posi- tionen der Gegenpartei, die den extensiven Bilderkult ablehnte, können entsprechend nur mühsam und ansatzweise rekonstruiert werden. Das ist dem Verfasser natürlich auch bewusst. Dennoch fügte er sich in sein vermeintliches Schicksal und benutzte mehr oder weniger kritisch die erhaltenen (gedruckten) Quellen.

Wie fast alle seine Vorgänger drückt sich auch Thümmel um die den Historiker ganz besonders interessierende Frage herum: Wieso stritt man sich um die Bilder und die Art und Weise ihrer Verehrung? Was war der Anlass dieser Auseinandersetzung? Man erfährt (45 ff.) eine schlichte Nacherzählung der einzigen relevanten Quelle, die darüber berichtet, der Chronographia des Theophanes aus dem beginnenden 9. Jh., fast ein Jahrhundert nach den geschilderten Ereignissen verfasst, in vielerlei Hinsichten (Quellen, Quellenbenutzung, Tendenz usw.) heftig umstritten, offenkundig eine "Meistererzählung « von Ereignissen, die heute nicht mehr verifiziert werden können (und vermutlich seit dem 9. Jh. nicht mehr [oder kaum] konnten), ein Paradebeispiel historischer Manipulation, die auch noch mehr als tausend Jahre nach der Abfassung das Bild von der Geschichte einer bestimmten Periode dominiert. ${ }^{7}$ Dagegen verblassen alle Geschichtsmanipulationen des I9. oder 20. Jahrhunderts!

Warum aber nahm (angeblich) die Verehrung von "Bildern « derart zu, dass sich Kaiser Leon III. veranlasst sah, dagegen einzuschreiten? Vielleicht allein deshalb, weil die umstürzenden Ereignisse des 7. Jahrhunderts, insbesondere die gigantische Krise des Staates und der sie tragenden Ideologien, verursacht durch die scheinbar unaufhaltsame Expansion des Islam und den dadurch ausgelösten radikalen Umbau des Staates, eine allgemeine Unsicherheit erzeugten, in der Individuen nach »Sicherheit« suchten? 
Jedoch kam es zu ikonoklastischen Maßnahmen gerade zu der Zeit - nach der erfolgreichen Abwehr des letzten, höchst gefährlichen Angriffs auf Konstantinopel 717/718 durch die Araber -, als die Situation des Reiches (relativ) gesichert war. Und den Höhepunkt - zur Zeit Kaiser Konstantins V. - erreichten die antiikonoklastischen Maßnahmen eben in dem Moment, in der sich Staat und Wirtschaft rapide erholten, die Steuereinnahmen stiegen und das Reich bedeutende militärische Erfolge erringen konnte. War der Ikonoklasmus also, salopp gesagt, ein gesellschaftsgeschichtliches »Luxusproblem «? 8 Diese Frage zu stellen ist angebracht, zumal ihre Beantwortung die Beurteilung des hier besprochenen Buches wesentlich beeinflusst.

Leider repräsentiert der Band nicht in jedem Punkt den neuesten Stand der Forschung. Wahrscheinlich war das dem Verfasser auch bewusst, allerdings ist seine Aussage (I I Anm. 2I) "Wenn eine Reihe von Arbeiten in der vorliegenden Darstellung fehlt, so habe ich dies zumeist in Rezensionen begründet «, nicht geeignet, diesen Mangel zu erklären. Das hat er nämlich nicht getan.

Dies betrifft die zentrale Monographie von Leslie Brubaker und John Haldon, Byzantium in the Iconoclast era (ca. 680-850): the Sources (Birmingham Byzantine and Ottoman monographs, 7), Aldershot 200I. Dieser Band hätte benutzt werden müssen! Und dies nicht nur, um einige völlig veraltete Ansichten zur Quellenproblematik (siehe das Kapitel »Die Quellen « I7-I9 und an vielen Orten) zu verhüten. Auch die umfänglich eingeleitete und ausführlich kommentierte englische Übersetzung der Chronographia des Theophanes von Cyril Mango und Roger Scott (The Chronicle of Theophanes Confessor. Byzantine and Near Eastern History AD 284-8I3. Translated with introduction and comment by C. Mango and R. Scott, Oxford I997) wurde ignoriert. Das ist nicht nur ein Schönheitsfehler, sondern führt bei der Bewertung der Informationen dieser zentralen Quelle oft zu Fehldeutungen. Die anhaltende Debatte um die Quellen des Theophanes - insbesondere die Rolle des Theophilos von Edessa - ist gänzlich an Thümmel vorbeigegangen. ${ }^{9}$ Auf S. 40 Anm. I4 I wird zur Quellenproblematik der Chronik des Theophanes allein auf Krumbachers » Geschichte der byzantinischen Litteratur « von I 897 verwiesen! Das ist schlicht peinlich und demonstriert die geäußerten Monita.

Leider fehlt auch ein bereits I999 (!) edierter Text, (angeblich) des Patriarchen Germanos, der Berücksichtigung hätte finden müssen. ${ }^{\text {Io }}$ Falls diese (nur georgisch erhaltene) Homilie »Über das Kreuz und die Bilder « tatsächlich Germanos zuzuweisen ist, was m. E. keineswegs sicher ist, sind einige Details des Ausbruchs des Bilderstreites vor (!) 730 in einem neuen Licht zu sehen. Falls der Text später (in den 6oer Jahren des 8. Jahrhunderts) entstand, bleibt er eine wichtige Quelle für die erste Phase des Bilderstreites. $\mathrm{Zu}$ beachten sind Anspielungen auf einen »ikonoklastischen « Eid, der den Gläubigen, die Bilder verehrten, abgefordert worden sein soll. ${ }^{\text {II }}$ Wenn dies stimmt, ist die gegen die Bilderverehrung gerichtete Politik Leons III. neu zu bewerten! Es müsste ihm dann doch eine intensivere bilderfeindliche Politik unterstellt werden, als vom Verfasser und der sonstigen Forschung angenommen wird. Vor diesem Hintergrund wird dann vielleicht auch der - zweifellos übertrieben erscheinende - Bericht des Liber Pontificalis (in der Vita des Papstes Gregor II., † 73I), dass bereits Leon III. (7I7-740) die Konstantinopolitaner gezwungen habe, die Verehrung der Ikonen Christi (nebst der Mutter Gottes und »aller Heiliger «) einzustellen, verständlicher, ${ }^{\mathrm{I} 2}$ auch
8 Zum wirtschaftlichen Aufschwung unter den ikonoklastischen Kaisern des 8. Jahrhunderts siehe Wolfram Brandes, Finanzverwaltung in Krisenzeiten, Frankfurt am Main 2002 sowie ERIC Ivison, Urban renewal and imperial revival in Byzantium (730I025), in: Byzantinische Forschungen 26 (2000) I-46.

9 Siehe eben Anm. 7. io Michel van Esbroeck, Un discours inédit de Germain de Constantinople sur la Croix et les Icônes, in: Orientalia Christiana Periodica 65 (I999) I9-5I (Edition und Übersetzung [S. 32-5I]). i I Van Esbroecck a.a. O. 46/48.

I2 Le Liber pontificalis, ed. Lours Duchesne, I, Paris I 886, 409. 
wenn der exakte Wortlaut des Papstbuches m. E. interpoliert ist. ${ }^{\mathbf{3} 3}$ Möglich jedoch - und vermutlich wahrscheinlicher - ist eine Entstehung des Textes in der Zeit um 765, als Kaiser Konstantin V., der Erzikonoklast, allen seinen Untertanen befahl, einen Eid abzulegen, nie eine Ikone zu verehren. Der Patriarch Konstantin II. musste dies vom Ambo der Hagia Sophia herab ebenfalls schwören. ${ }^{\mathrm{I}}{ }^{\mathrm{D}}$ Diese Praxis machte Schule und im Jahre 780 musste Patriarch Paulos IV. ebenso schwören. ${ }^{15}$ Der Verfasser betont zwar verschiedentlich, dass die Bischöfe, die am Nicaenum II teilnahmen, sämtlich ein gegen den Bilderkult gerichtetes Glaubensbekenntnis unterschrieben haben mussten (siehe z. B. II9, I 24, I 28 usw.), lässt sich jedoch die Erörterung dieser Eide entgehen. Die Vereidigung der gesamten Bevölkerung zu einer Frage, die direkt zum Gegenstand dieses Buches gehört, hätte unbedingt behandelt werden müssen. Hier galt es kein Neuland zu beschreiten, denn die byzantinischen Untertaneneide, ein Erbe der Spätantike, sind umfassend untersucht. ${ }^{\mathbf{6}}$ Ist es mithin ein Zufall, dass eben die Kaiserin Eirene, die die Bilderverehrung 787 wieder einführte, ein Gesetz über den Eid erließ? Zwar geht es hier um die Rolle von Eiden bei alltäglichen Rechtsgeschäften und in Prozessen, doch sagt das Proömion - der Ort eines Gesetzes, wo die Kaiser generelle politische Absichten und Konzepte äußerten (oft auch unabhängig vom eigentlichen Gegenstand des Gesetzes) - ausdrücklich, das Matthäusevangelium $(5,34)$ zitierend, man solle überhaupt nicht schwören: "... denn aus dem Eid entsteht Meineidigkeit, und die bedeutet Gottesleugnung ${ }{ }^{\mathbf{I}}{ }^{\mathbf{7}}$ Auch diese Novelle ist m. E. im Kontext der von den ikonoklastischen Kaisern für die Durchsetzung ihrer Politik eingesetzten Untertaneneide zu sehen. All dies gehört in die Schilderung der gesellschaftlichen Verortung des Bilderstreites und der in dieser Zeit in Byzanz veranstalteten Synoden!

Ein großes Problem (neben den oben erwähnten zahlreichen Arbeiten von Paul Speck) kam auf den Verfasser zu, als vor etlichen Jahren Erich Lamberz (Arbeitsstelle der Acta Conciliorum Oecumenicorum an der Bayerischen Akademie) begann, die Akten des 7. Ökumenischen Konzils von 787 neu zu edieren. Thümmel musste allerdings meist noch nach der veralteten und an vielen Stellen unrichtigen Ausgabe von Mansi (Bände XII-XIII, I766/I776) zitieren. Lamberz veröffentlichte in den letzten Jahren verschiedene zentrale Arbeiten - in Vorbereitung der Edition bzw. deren Fortschritte dokumentierend -, die zwar alle von Thümmel aufgelistet werden (XVI), jedoch in vielen Fällen nicht wirklich verarbeitet wurden. Es war sicher richtig und ist dem Verfasser nicht negativ anzurechnen, dass er diese Edition nicht abwartete und sein Buch bereits jetzt veröffentlichte. Und es ist nachvollziehbar, dass es mehr als ärgerlich ist, wenn ein nahezu abgeschlossenes Manuskript unentwegt durch neue Artikel - zumindest partiell - in Frage gestellt wird. Dennoch ist hier ein Manko anzumerken.

Da Lamberz wie kein anderer die handschriftliche Überlieferung kennt und mit gleichsam kriminalistischen Fähigkeiten ausgestattet ist, hat er nicht nur zahlreiche Textverbesserungen publiziert (man darf mithin auf die vollständige Edition der umfangreichen Akten gespannt sein), er hat auch eine Anzahl wichtiger Textpassagen, die in der historischen Diskussion eine zentrale Rolle spielten, grundlegend neu bewertet.

Eine nicht unerhebliche Frage ist die nach der Historizität einer 8. Sitzung des Konzils, angeblich im Magnaurapalast in Konstantinopel abgehalten. Lamberz bezweifelte dies mit Ver-

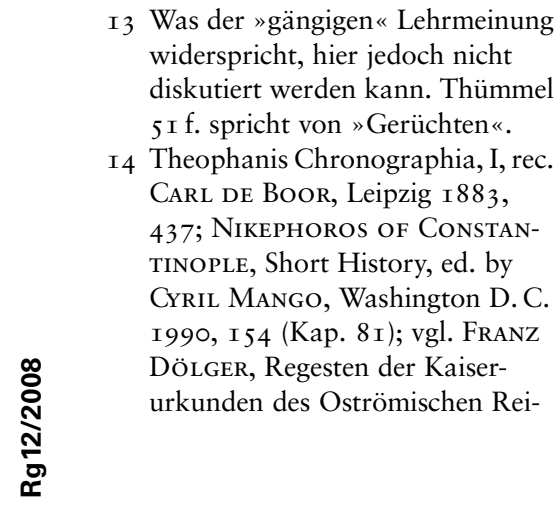

Brandes, Bilder und Synoden ches, I, München I924, Nr. 324. Auch in hagiographischen Quellen erwähnt!

I 5 Theoph. 457 DE Boor; vgl. Venance Grumel/Jean DarROUZÈs, Régestes des actes du patriarcat de Constantinople, $\mathrm{I} / 2-3$, Paris I989, Nr. 348 .

I6 Nicolas Svoronos, Le serment de fidélité à l'empereur byzantin et sa signification constitutionelle, in: Revue des études byzantines 9 (I95I) I06-I42.

I7 Ludwig Burgmann, Die Novellen der Kaiserin Eirene, in: Fontes Minores IV (I98I) I-36, hier I6/ I 8 (Übersetzung nach Burgmann). 
weis auf die handschriftliche Überlieferung. ${ }^{\text {I8 }}$ Thümmel hingegen hält - Lamberz' Überlegungen explizit zurückweisend ${ }^{\text {I9 }}$ - an der Historizität fest. Dabei ist klar, dass bereits Anastasius Bibliothecarius, der berühmte römische Übersetzer, als er ca. 870 eine lateinische Übersetzung der Akten anfertigte, eine griechische Vorlage hatte, die nur über sieben Sitzungen verfügte. Dagegen argumentierte Thümmel (92 ff., I 79 f.). Unter anderem meinte er, dass Anastasius nicht wie man bisher einhellig annahm (und was m. E. auch völlig zutreffend ist) - einen griechischen Kodex aus Konstantinopel mit nach Rom brachte, wo er ihn dann übersetzte, sondern dass er einen in Rom befindlichen - nach Thümmel den nach 787 dorthin gesandten Text der Konzilsakten - Kodex benutzte. Dies ist m. E. unmöglich, denn Lamberz hat bei der Behandlung zweier Papstbriefe, die in den Akten enthalten sind, unwiderlegbar gezeigt, dass diese noch zwischen 860 und $87 \mathrm{I}$ in Konstantinopel manipuliert worden sind (dazu gleich). ${ }^{2 \circ} \mathrm{Da}$ Anastasius gerade diese Manipulationen bemerkte und sich ausführlich darüber ausließ, muss er einen Kodex vor sich gehabt haben, der frühestens nach 860 entstand. Damit sind Thümmels Überlegungen hinfällig. ${ }^{2 \mathrm{I}}$

Der Rechtshistoriker stößt sich an der lieblosen (oder doch ignoranten?) Behandlung juristischer, kanonistischer Probleme. Was versteht der Verfasser unter »dogmatisch-kirchenrechtlichen Kompendien «? Genannt werden im Abschnitt über die Quellen (I I Anm. 22) lediglich Euthymios Zigabenos, Niketas Choniates, Matthaios Blastares. Und was ist mit dem Nomokanon in 14 Titeln oder der Synagoga in 50 Titeln des Ioannes Scholastikos (von den großen Kanonisten Zonaras oder Balsamon abgesehen) sowie diversen anderen Kanonessammlungen? Zur Zeit des Konzils (787) lagen insbesondere der
Nomokanon in I4 Titeln und die Synagoga in 50 Titeln vor. Eine Analyse der Konzilsakten hat mithin der Frage nachzugehen, ob diese weit verbreiteten Handbücher Verwendung fanden. Deutlich wird dies, z. B. in Thümmels Behandlung der I. und auch der 2. Sitzung (I33-I47). Hier ging es u.a. darum, wie mit häretischen Bischöfen zu verfahren sei. Sollten sie mit oder ohne Buße wieder ihre Positionen einnehmen dürfen? Wie könnte eine akzeptable Buße aussehen? Thümmel unterstellt ein "vorbereitetes Dossier « (I37) und denkt mehrfach an eine umfangreiche Vorbereitungstätigkeit der patriarchalen Bibliothekare. Nun ist sicher nicht abzustreiten, dass der Patriarch Tarasios das ganze Konzil sehr gut vorbereitet hat und alle möglichen Details inszeniert erscheinen. Doch was diese kanonistischen Probleme betrifft, mussten sich die zuständigen Personen (Chartophylax, Bibliophylax, Skeuophylax ${ }^{22}$ ) im vorliegenden Falle keineswegs in staubige Bände vertiefen. Ein Blick in die Synagoga in 50 Titeln - ich gehe davon aus, dass diese benutzt wurde, wie die Reihenfolge der herangezogenen Kanones nahelegt - hat sicher genügt. Daraus eine große Aktion zu machen, zeugt von wenig Kenntnis der kanonistischen Quellen.

Auch die Behandlung der Kanones, die dieses Konzil erließ, ${ }^{23}$ ist sehr unbefriedigend (I 84I90). Allein schon der Umstand, dass auch die Kanones allein nach Mansi zitiert werden, stimmt bedenklich. Eine Einordnung in die Geschichte des kanonischen Rechts fehlt völlig. Geboten wird einzig eine (an einigen Stellen fragwürdige) Inhaltsangabe ausgewählter Kanones.

Es lassen sich zahlreiche weitere Fehler im Detail, manchmal auch mit weitreichenden Konsequenzen, auflisten. Oft sind es simple Fehler im Bereich des Handwerklichen, über die man stolpert. Grundlegende Hilfsmittel (wie die Patriar-
I 8 ERICH LAmberz, Die Bischofslisten des VII. Ökumenischen Konzils (Nicaenum II), München 2004, $28 \mathrm{f}$, $35 \mathrm{f}$.

I9 So auch in Thümmels Rezension von Lamberz, Die Bischofslisten (wie Anm. I8), in: Annuarium Historiae Conciliorum 36 (2004) $243 \mathrm{ff}$.

20 LAMBERZ, »Falsata Graecorum more «? ..., in: Novum Millennium (wie Anm. 4), 213-229.
2I Gegen Thümmel spricht auch der riesige logistische Aufwand und die anormale Reihenfolge der Kaisernamen bei den Akklamationen der sog. 8. Sitzung (I.-7. Sitzung: Konstantin, dann Eirene; 8. Sitzung: Eirene, dann Konstantin).

22 S. I32 werden diese aufgelistet, wenn auch höchst fragwürdig. $\mathrm{Zu}$ den einzelnen Funktionen hätte einfach JEAN DARrouZÈs,
Recherches sur les Offikia de l'église byzantine, Paris I970 benutzt werden können, um so dumme Fehler zu vermeiden.

23 Jetzt kritisch ediert von ERICH LAMBERZ, in: Conciliorum oecumenicorum generaliumque decreta, I: The Oecumenical Councils, Turnhout 2006, 317-344. 
chatsregesten von Grumel und Darrouzès, die »Prosopographie der mittelbyzantinischen Zeit « [= PmbZ] oder die CD-ROM der »Prosopography of the Byzantine Empire « [= PBE]) wurden nicht benutzt, obwohl sie im Literaturverzeichnis auftauchen. Das führt gelegentlich zu eigenartigen Aussagen. So etwa 236 Anm. I I 29, wo bemerkt wird, dass der Erzbischof Theophil nur aus einer Quelle (der Vita Euthymii) bekannt sei, während die PmbZ Nr. 8209 diverse weitere Quellen und eine Biographie des Theophil bietet (derartige Beispiele gibt es zahlreich; man vgl. nur die I 3 I f. aufgelisteten »Hilfskräfte « bei der Abhaltung des Konzils mit den jeweiligen Lemmata der PmbZ oder der PBE). Eine gewisse Unvertrautheit mit der byzantinischen Verwaltung wird da deutlich, wo der Verfasser etwa die kaiserlichen Vertreter am Konzil benennt. Es ist immer sehr gefährlich, byzantinische Amtsbezeichnungen mit modernen Titeln zu übersetzen (so besonders I $28 \mathrm{f}$.). Es sind diese zahllosen Fehler, missverständliche Formulierungen, längst überholte Ansichten usw., die es dem Byzanzhistoriker schwer machen, den Band zufrieden unter die oft benutzten Handbücher einzureihen. Wer auf eine wirkliche Verortung des Geschehens gerechnet hat, sieht sich enttäuscht. Man muss also auf die lange angekündigte Mono-

\section{Lost law?*}

If a book with such a theme had been published hundred years ago, it would have been widely read among legal scholars in the Nordic countries. Looking back into the central journal of Nordic legal science - Tidsskrift for Retsvidenskab - piles of books dealing with Germanic graphie von John Haldon und Leslie Brubaker über den Bilderstreit warten. Die z.T. gravierenden Differenzen zu Thümmels Darstellung seien schon an dieser Stelle annonciert. ${ }^{24}$ Ich habe diese hier nicht angeführt und verweise den Leser/die Leserin auf dieses hoffentlich bald erscheinende Buch.

Nach einigen Seiten heftiger Kritik sollen jedoch auch einige Vorzüge des Bandes benannt werden. Es ist zweifellos für alle geeignet, die schnell einen Überblick über die wichtigsten Ereignisse der Kirchengeschichte während des sog. Bilderstreites im 8. und 9. Jahrhundert suchen. Die Entwicklung der theologischen Positionen und die zunehmende Verfeinerung der theologischen Argumentation für den Bilderkult, der bis heute einen zentralen Bestandteil des Selbstverständnisses der orthodoxen Kirchen darstellt, werden anschaulich verdeutlicht. ${ }^{25}$

Und dennoch bleibt ein bitterer Beigeschmack. Angesichts des Umstandes, dass dieser Band in einer so zentralen Reihe erschienen ist und sicher bald in diverse Sprachen übersetzt werden wird, werden seine Fehler und Mängel in der nächsten Zeit verbreitet werden. Daran wird auch eine kritische Rezension nichts ändern.

Wolfram Brandes themes were discussed as part of the ongoing conceptualisation of legal science and legal ideology. The cultural shift from Romanism to Germanism in the decades around I900 influenced many aspects of legal science and the general image of law in society. But from the Nordic
24 John Haldon war so freundlich, mir wesentliche Teile des ungedruckten Manuskripts zuzusenden.

25 Auch wenn gelegentliche (XXIf.) Ausfälle gegen HeInz OHME, Ikonen, historische Kritik und Traditio, in: Zeitschrift für Kirchengeschichte I IO (I999) I-24 befremden.

\footnotetext{
* Gerhard Dilcher, Eva-Marie Distler (Hg.), Leges - Gentes Regna. Zur Rolle von germanischen Rechtsgewohnheiten und lateinischer Schrifttradition bei der Ausbildung der frühmittalterlichen Rechtskultur. Berlin: Erich Schmidt Verlag 2006, 650 S., ISBN 978-3-503-07973-5
} 